\title{
STATUS of SHORT-PULSE KrF AMPLIFIER RESEARCH and DEVELOPMENT at HILL, SZEGED
}

\author{
S. Szatmári ${ }^{1,2 *}$, Z. Szántóo ${ }^{2}$ R. Bognár ${ }^{2}$, R. Dajka ${ }^{2,3}$, I. B. Földes ${ }^{2,4}$ \\ ${ }^{1}$ Department of Experimental Physics, University of Szeged, Dóm tér 9, H-6720 Szeged, Hungary \\ ${ }^{2}$ Institute of Physics, University of Szeged, Dóm tér 9, H-6720 Szeged, Hungary \\ ${ }^{3}$ Department of Photonics and Laser Research, Interdisciplinary Excellence Centre, University of \\ Szeged, Dugonics tér 13, H-6720 Szeged, Hungary \\ ${ }^{4}$ Department of High Energy Experimental Particle and Heavy Ion Physics, Wigner Research Centre \\ for Physics, H-1121 Budapest,Hungary
}

DOI: https://doi.org/10.14232/kvantumelektronika.9.32

\begin{abstract}
The small saturation energy density of excimers requires amplifiers of large cross-sections for amplification of short pulses of already medium power. Homogeneous excitation of large volumes of Fluorine-based gas mixtures by discharge pumping is a critical interplay of the properties of both pumping and preionization; generally necessitating an intense, spatially and temporally controlled xray preionization.

In the present realization at High Intensity Laser Laboratory (HILL) the stringent intensity requirements of preionization are fulfilled by reducing the pulse duration of the x-ray flash to $\sim 16 \mathrm{~ns}$, and by positioning the $\mathrm{x}$-ray source in the near vicinity of the active volume. By proper choice of the positions of two cylindrical x-ray guns the spatial distribution of preionization can be tuned to (and around) the optimum distribution giving a practical method to compensate for eventual inhomogenities of the E-field of excitation and to tune the discharge to the desired geometry. In this way the realization of a $\mathrm{KrF}$ excimer amplifier of $\sim 5 \mathrm{x} 4 \mathrm{~cm}^{2}$ cross-section is presented.
\end{abstract}

\section{Introduction}

In the view of the recent progress of IR solid-state laser systems high-brightness ultraviolet (UV) excimer lasers can be regarded as complementary sources. Their main advantage occurs mainly in those experiments where high photon energy, optimum spatial concentration and/or efficient conversion of the pulse energy to radiation of even shorter wavelength are needed [1,2]. At present the maximum peak power of short-pulse excimer systems is limited to the TW level $[1,2,4,5]$ by the difficulties associated with the construction of short-pulse UV amplifiers $[1,2]$ and by the inherently limited energy extraction from excimer amplifiers of short energy storage time $[1,3]$.

Excimers are ideal four-level systems allowing very efficient operation even in the UV for pulses longer than the storage (or pumping) time. However, they exhibit moderate extraction efficiency for shorter pulses, because of the relatively short (several ns) storage time compared to the accessible pumping times (several times $10 \mathrm{~ns}$, or more).

The saturation energy density of excimers is very low compared to solid-state systems; typically is in the range of several $\mathrm{mJ} / \mathrm{cm}^{2}$. In KrF power amplifiers the optimum operation both for efficiency and contrast is a critical function of the energy density $[1,6]$, which can only be maintained when the energy density is set to $\varepsilon_{\mathrm{opt}} \approx 2.2 \mathrm{x} \varepsilon_{\mathrm{sat}} \approx 4.5 \mathrm{~mJ} / \mathrm{cm}^{2}[1,2,6,7]$. This condition requires large amplifier cross-sections already for moderate output energies.

Due to these requirements, pumping of excimer gain modules can only be realized by an efficient and temporally short pumping mechanism capable of homogeneously excite a large volume of large crosssection in a short time comparable to the energy storage time. Discharge pumping of excimers is more 
straightforward for many practical reasons, however special considerations and corresponding steps are needed to homogeneously deposit the electric energy $[4,5,9,10]$.

It is known from former gain dynamic studies [1,8], that it is only $\mathrm{KrF}$ and $\mathrm{ArF}$ which acts as a real four-level system when short-pulse amplification is needed, allowing good access to the momentarily stored energy. On the other hand these are just the excimers where homogeneous excitation of the initial Fluorine-based gas mixture is the most critical; special measures are needed to ensure arc-free, homogeneous excitation of large volumes [11]. One needs a fast rising, temporally clean electric pulse to produce a highly uniform electric field together with a fast and efficient preionization of the gas characterized by a similarly uniform spatial distribution of the generated electrons (ions).

From the optical point of view a circular cross-section is ideal, necessitating a discharge cross-section of $w / d \approx 1$ aspect ratio (where $w$ is the width of the discharge, $\mathrm{d}$ is the separation between the electrodes). A discharge of large cross section and of aspect ratio, however, necessitates the use of $\mathrm{x}$ ray preionization which is known to be capable of homogeneously ionizing large volumes [9, 11-14]. The large penetration depth (small absorption coefficient) of the x-ray radiation in the gas mixture is a necessary condition to achieve spatially uniform ionization. The small absorption, however, minimizes the absorbed energy. This sets stringent requirements to the strength of the x-ray source. Because of the very fast (a few times $10 \mathrm{~ns}$ ) recombination of the electrons in the Fluorine-based gas mixtures, preionization must be comparably fast, necessitating an intense x-ray source of similar or shorter duration precisely synchronized to the main pumping pulse. A possible way to increase the intensity of x-rays is to decrease the spatial separation of the x-ray source and the gas volume to be preionized. This, however, generally leads to a spatially inhomogeneous intensity distribution.

Former investigations showed that the spatial distribution of the discharge is the critical interplay of that of both the E-field and the preionization [15]. For a given E-field distribution this makes, in principle possible to fine-tune the discharge geometry by controlling the spatial characteristics of preionization.

Based on the geometrical requirements imposed by the planned volume of $\sim 40 \times 5 \times 5 \mathrm{~cm}^{3}$ to be preionized, and by our former experimental experience associated with the development of pencillike, pulsed, x-ray sources of reflective geometry, in this paper design considerations and first results are reported for a large aperture, short-pulse $\mathrm{KrF}$ amplifier module, preionized by a spatially-tunable, twin $\mathrm{x}$-ray gun.

\section{Experimental realization}

\section{1. $x$-ray preionizer/driving circuit}

As it is described in the Introduction, a spatially elongated, intense $x$-ray source of short pulse duration is mandatory for efficient preionization of a transversally pumped $\mathrm{KrF}$ excimer gas mixture. For practical reasons (described later) our development was concentrated on the realization of a twin, cylindrical $x$-ray gun powered by a common power supply. With regard to the pulsed operation required here a pulsed plasma electron source was chosen as the (cold) cathode of the x-ray gun [16]. Among the possible cathode designs the so-called carbon-epoxy capillary cathode design was selected [17]. The realization of the x-ray gun is shown in Fig. 1. The diode is formed by a cylindrical tungsten anode of $6.3 \mathrm{~mm}$ diameter and $50 \mathrm{~cm}$ length, positioned in the centre of a glass tube of $40 \mathrm{~mm}$ diameter and $1.5 \mathrm{~mm}$ wall thickness. This anode is supported at both ends of the tube by insulators far away from the ends of the somewhat shorter cathode of $\sim 40 \mathrm{~cm}$ length. The cathode is an array of carbon-epoxy capillaries (Van Dijk Pultrusion Products). Detailed structure of the coldcathode diode of the x-ray gun is also shown in Fig. 1; by the enlarged - side and cross-sectional view of the central portion of the tube. The anode is fed by a $\sim 100 \mathrm{kV}$ positive voltage pulse at one end of the evacuated tube, which is directly connected to an oil-filled cylindrical pulse forming line (which connects at the same time the x-ray gun to the power supply). 


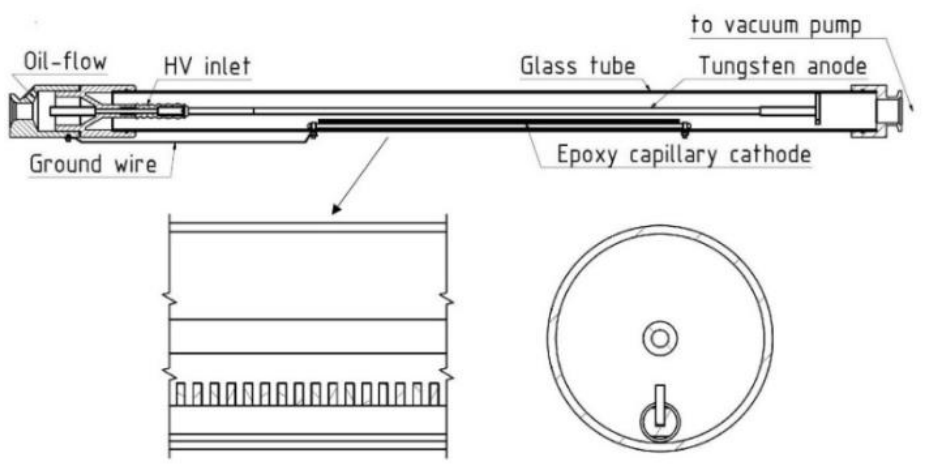

Figure 1 Schematics of the cold-cathode $x$-ray gun of cylindrical geometry

A special pulsed power supply was developed, which could drive both diodes by a synchronized $>100 \mathrm{kV}$ electric pulses of $\sim 30 \mathrm{~ns}$ duration, and of $\sim 20 \mathrm{~ns}$ risetime, as shown by curve U in Fig. 2. Curve I is the corresponding current, measured at the same point by a Rogowski pick-up coil.

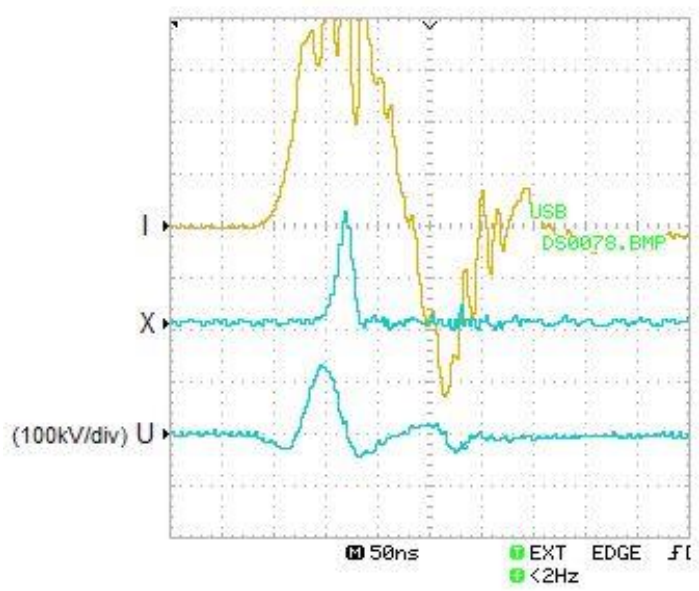

Figure 2 Temporal behavior of the current (I), the voltage $(U)$ and the $x$-ray pulse $(X)$ of the preionizer

The dose and the temporal behavior of the x-ray radiation generated by one diode was determined by an ionization chamber and by a fast silicon-diode amplifier arrangement, both positioned $10 \mathrm{~cm}$ away from the cylindrical anode of the x-ray gun. The x-ray dose (for one x-ray gun) was measured to $11 \mathrm{mR}$ and the FWHM of the x-ray pulse was $16 \mathrm{~ns}$. The oscilloscope trace of the latter measurement is shown in Fig. 2 by curve $\mathrm{X}$. The $16 \mathrm{~ns}$ temporal width for the $\mathrm{x}$-ray pulse is obtained through deconvolution of the measured value by the $10 \mathrm{~ns}$ resolution of the detection system. Note, that the very short duration of $\mathrm{x}$-rays compared to the former approaches is a real advantage in minimizing the necessary electric energy, while reaching even larger electron density. It means that using a short $(20 \mathrm{~ns}) \mathrm{x}$-ray pulse (of the same, $30 \mathrm{mR}$ dose) an electron density of $3 \times 10^{8} 1 / \mathrm{cm}^{3}$ and $4 \times 10^{8} 1 / \mathrm{cm}^{3}$ can be reached for $\mathrm{Xe} / \mathrm{He}$ and $\mathrm{Xe} / \mathrm{Ne}$-based Fluorine gas mixtures. On the other hand, if one uses a $400 \mathrm{~ns}$ long X-ray pulse (as in many former experiments) the electron density remains definitely below $10^{7} 1 / \mathrm{cm}^{3}$.

\section{Discharge chamber / charging scheme}

Our aim was to homogeneously pump a $40 \times 5 \times 5 \mathrm{~cm}^{3}$ volume of a $\mathrm{KrF}$ gas mix. The cross-sectional view of the discharge chamber accommodating to this condition is shown in Fig. 3. The upper (cathode) and lower (anode) electrodes together with the electrode-holder plates are made of aluminum. The cathode is a flat electrode, while the anode is designed to homogeneously pump (to ensure homogeneous E-field distribution over) a $5 \times 5 \mathrm{~cm}^{2}$ region (marked by dashed lines). The insulator - forming the side walls of the chamber - is made of PVDF. Twin X-ray tubes are indicated in Fig. 3 (marked by X) for preionization through the cathode. In order to make the discharge area "accessible" by x-rays, windows are milled out on the upper electrode and on the electrode holder plate. The geometry of these windows is also seen in Fig. 3, allowing the irradiation of the pumped volume by two x-ray guns in a large solid angle. The remaining thickness of the windows is $0.7 \mathrm{~mm}$. 


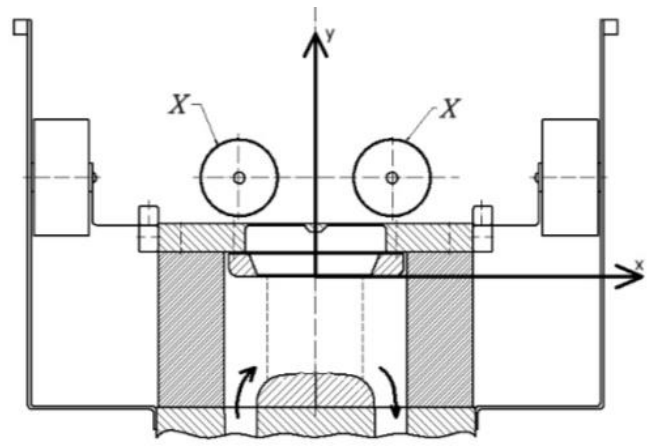

Figure 3 Cross-sectional view of the discharge chamber allowing $x$-ray preionizations by two $x$-ray guns $(X)$.

The laser chamber was connected to a gas reservoir/circulating fan (Lambda Physik Göttingen $\mathrm{GmbH}$ ) allowing efficient transversal gas circulation in the chamber (indicated by arrows in the Figure), thus promoting high rep-rate operation.

In order to identify the different positions of the preionizers, a coordinate-system - centered to the axis of the cathode electrode as used in our calculations - is also shown in the Figure. For the characterization of the positions of the x-ray guns - arranged always symmetrically to the $\mathrm{y}$ axis their horizontal spatial separation $(\Delta x=2 x)$ and their $y$ coordinates are used (for further details see next section).

The electric charging circuit - shown in Fig. 4 - is a standard, thyratron (CX1573C, E2V Technologies) driven L-C inversion circuit, completed by a magnetic switch compressor (MSC) technology. For the given value of the main capacitor bank $\left(C_{1}=C_{2}=96 \mathrm{nF}\right.$, determined by the electric energy to be transferred) the inversion time is synchronized to the switching of MSC by proper choice of L and of the cross-section of the core of the MSC (Metglas 2605 Co) surrounded by a "spatially distributed coil" of 1 winding. The peaking capacitance $\mathrm{C}_{3}=50 \mathrm{nF}$ is equally distributed along the two sides of the discharge chamber (see also Fig. 3). With the use of this charging circuit a $60 \mathrm{kV}$ electric pulse of $120 \mathrm{~ns}$ risetime could be produced on the cathode electrode, at a $\mathrm{U}_{0}=32 \mathrm{kV}$ power supply voltage.

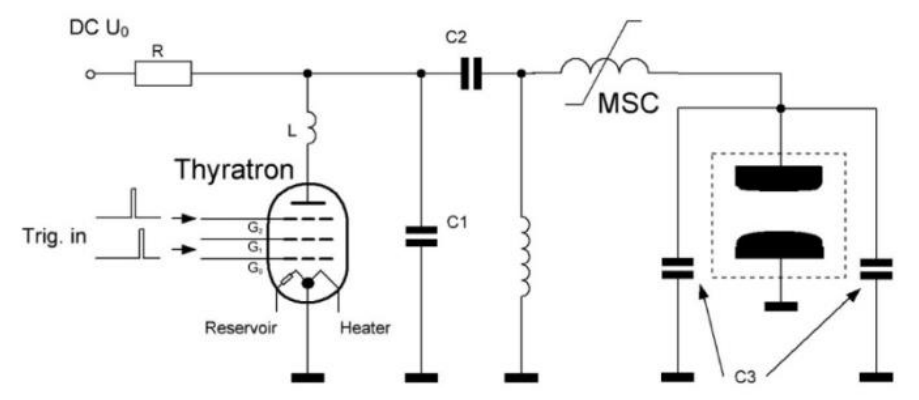

Figure 4 Schematics of the L-C inversion and magnetic switch compressor (MSC) based charging circuit

Temporal behavior of this pulse (curve U) is shown in Fig. 5 at $\mathrm{U}_{0}=30 \mathrm{kV}$. Curve I is the discharge current, which is picked up by pick up coil, inserted into the discharge loop.

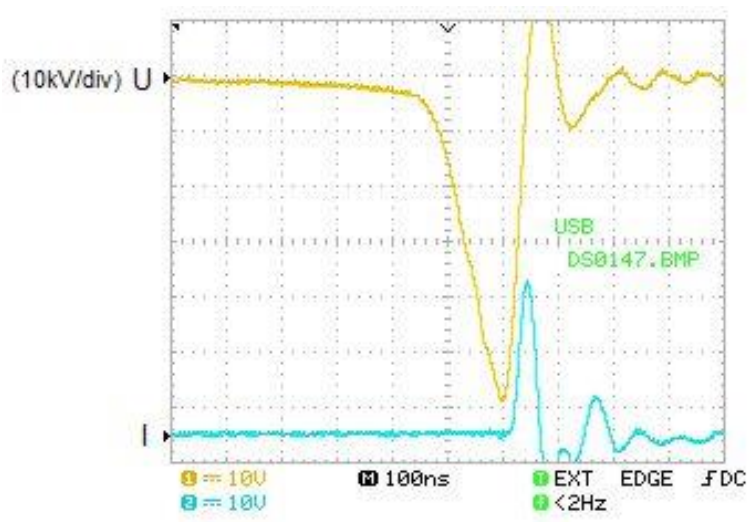

Figure 5 Temporal behavior of the electric pulse measured on the cathode electrode (curve $U$ ) and of the discharge current (curve I) 
The short duration of the preionization process and that of the main discharge requires exact synchronization of the two charging circuits, including the compensation of their long-term drift (mainly initiated by the thyratrons). For this purpose an automatic synchronization unit - based on optical fiber communication - was developed, to compensate for the long-term drift, in $5 \mathrm{~ns}$ steps. In the case of all the following comparative measurements the same gas mixtures were used (120 mbar He with 5\% F2, 150 mbar Kr filled up to 2.0 bar with $\mathrm{He}$ ).

\section{Results}

\section{Spatial distribution of the $x$-ray field of preionization}

In this section the results of our considerations, numerical calculations and of corresponding measurements for the spatial distribution of the x-ray intensity are presented. Since absorption of xrays in the $\mathrm{KrF}$ gas mixture is weak, its contribution to the spatial distribution was neglected. Based on the cylindrical emission geometry of the x-ray sources, $\mathrm{r}^{-1}$ spatial dependence of the $\mathrm{x}$-ray intensity was assumed/used in our calculations.

In Fig. 6 the calculated horizontal distribution of the $x$-ray intensity for a given $(\Delta x=80, y=45)$ position of the two cylindrical $\mathrm{x}$-ray sources is shown by solid lines in different horizontal planes thorough the discharge volume (for different values of $y$ ). The shape of the curves changes from convex to concave (through a flat) as y changes. The two dashed lines confine again that region, where homogeneous pumping is provided. Most homogeneous preionization seems to be best fulfilled in a plane characterized by $y=-10$ ( $10 \mathrm{~mm}$ below the cathode electrode).

All these calculations were confirmed by measurements; the spatial dependence of the x-ray intensity along the $\mathrm{x}$-axis was measured for different $\mathrm{y}$ values. This measurement clearly confirmed the results of calculations.

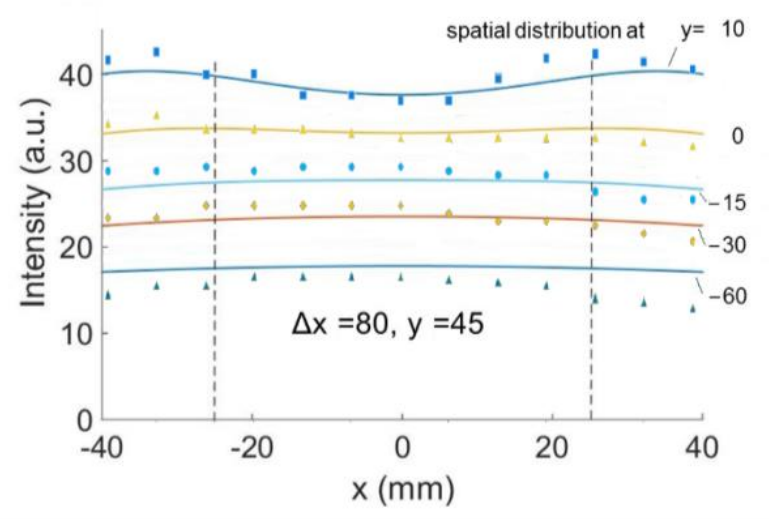

Figure 6 Calculated distribution of the $x$-ray field (solid lines) along the $x$ axis for different values of $y$ (when the position of the $x$-ray guns is $\Delta x=80$, $y=45)$. The points are measured values.

It is a reasonable assumption that along the electric field vectors in the pumped area (along the $y$ axis in Fig. 3) it is the integral (or the average value) of the x-ray field strength which determines the (integrated) effect of preionization for a fixed value of $x$. Performing such calculations, the resulting curves show that by changing (symmetrically) the horizontal positions of the x-ray sources (changing their $\Delta \mathrm{x}$ horizontal separation from $70 \mathrm{~mm}$ to $100 \mathrm{~mm}$ ) the "integrated" distribution can also be tuned to either concave or to convex. The main claim of these considerations is that using line-emitting $\mathrm{x}$ ray sources of cylindrical emission geometry, even in the near vicinity of the discharge volume, homogeneous (integrated) $\mathrm{x}$-ray distribution can be achieved along the axis perpendicular to the Efield. This offers to fulfill the most important necessary condition for homogeneous energy deposition by the discharge. Moreover, by changing the horizontal spatial separation of the x-ray sources, easy tuning can be realized; either the middle or the outer sections of the discharge volume are irradiated more intensively, which can compensate for eventual inhomogeneities of the E-field of excitation. In this way the desired discharge geometry can be produced in a technically simple way, which is of great practical importance. 
Under these optimized experimental conditions the spatial distribution of the emission of the $\mathrm{KrF}$ amplifier module was measured; in a free running oscillator mode the spatial distribution of the emission is shown in Fig. 7 indicating a homogeneous flat-topped distribution.

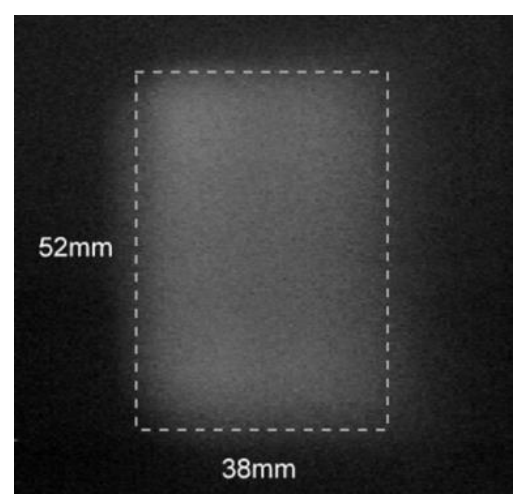

Figure 7 Spatial distribution of the output beam.

\section{Acknowledgements}

This work was supported by the European Social Fund EFOP-3.6.2-16-2017-00005 - Ultrafast physical processes in atoms, molecules, nanostructures and biological systems. We are grateful to the support (Hungary grant NKFIH-1279-2/2020-TKP2020 of Ministry of Innovation and Technology). The authors thank E. Müller-Horsche (Hochshule Augsburg), Ya. E. Krasik (Israel Institute of Technology) and G. Firla (VAC GmbH, Germany) for valuable discussions, B. Gilicze, B. Csánk and L. Gyihor for their participation in some parts of this R\&D activity.

\section{References}

[1] S. Szatmári, G. Marowsky and P. Simon, Landolt-Börnstein - Group VIII 1B1 (Eds. G. Herziger, H. Weber, R. Poprawe), 215 (2007). http://doi.org/10.1007/978-3-540-44821-1_5

[2] A. B. Borisov,C. J. McCorkindale, S. Poopalasingam, J. W. Longworth, P. Simon, S. Szatmári and C. K. Rhodes, Reports on Progress in Physics 79, 046401 (2016).

https://doi.org/10.1088/0034-4885/79/4/046401

[3] S. Szatmári, P. Simon, Optics Communications 98, 181 (1993). https://doi.org/10.1016/0030-4018(93)90777-3

[4] S. Szatmári, F.P. Schäfer, E. Müller-Horsche, W. Mückenheim, Optics Communications 63, 305 (1987). https://doi.org/10.1016/0030-4018(87)90181-7

[5] R. Nodomi ; Y. Oeda ; K. Sajiki ; S. Nakajima ; M. Watanabe ; S. Watanabe, IEEE Journal of Quantum Electronics 27, 441 (1991). https://doi.org/10.1109/3.81344

[6] G. Almási, S. Szatmári, P. Simon, Optics Communications 88, 231 (1992). https://doi.org/10.1016/0030-4018(92)90513-Q

[7] S. Szatmári, G. Almási, P. Simon, Applied Physics B 53, 82 (1991). https://doi.org/10.1007/BF00325535

[8] S. Szatmári, F.P. Schäfer, Journal of Optical Society of America B 4, 1943 (1987). https://doi.org/10.1364/JOSAB.4.001943

[9] H. Mizoguchi, A. Endoh, J. Jethwa, B. Rácz, F. P. Schäfer, Applied Physics B 52, 195 (1991). https://doi.org/10.1007/BF00750951 
[10] G. Kovács, S. Szatmári, Measurement Science and Technology 5, 127 (1994). https://doi.org/10.1088/0957-0233/5/2/008

[11] H. Shields, SPIE Proceedings 1046, 15 (1989). https://doi.org/10.1117/12.951330

[12] C. Tallman, SPIE Proceedings 1046, 2 (1989). https://doi.org/10.1117/12.951329

[13] K. Midorikawa, M. Obara, T. Fujioka, IEEE Journal of Quantum Electronics 20, 198 (1984). https://doi.org/10.1109/JQE.1984.1072388

[14] M. Steyer, Journal of Physics D: Applied Physics 23, 18 (1990). https://doi.org/10.1088/0022-3727/23/1/004

[15] M. S. Pronko, IEEE Journal of Quantum Electronics 30, 2147 (1994). https://doi.org/10.1109/3.309877

[16] Ya. E. Krasik, D. Yarmolich, J. Z. Gleizer, V. Vekselman, Y. Hadas, V. Tz. Gurovich, and J. Felsteiner, Physics of Plasmas 16, 057103 (2009). https://doi.org/10.1063/1.3085797

[17] J. Z. Gleizer, T. Queller, Yu. Bliokh, S. Yatom, V. Vekselman, Ya. E. Krasik, and V. Bernshtam, Journal of Applied Physics 112, 023303 (2012). https://doi.org/10.1063/1.4739258 